\title{
Anastomosing hemangioma simulating renal cell carcinoma
}

Mariana Athaniel Silva Rodrigues ${ }^{1}$, Eduardo Kaiser Ururahy Nunes Fonseca ${ }^{~}$, Fernando Ide Yamauchi ${ }^{1}$, Ronaldo Hueb Baroni ${ }^{1}$

${ }^{1}$ Departamento de Imagem, Hospital Israelita Albert Einstein, São Paulo, SP, Brasil

\section{ABSTRACT}

The anastomosing hemangioma is a recent described rare variant, which histologically simulates an angiosarcoma and occurs primarily in the genitourinary tract. We present a case of renal anastomosing hemangioma from a radiologic perspective, describing its imaging features and reviewing its presentation and management.

Keywords: Radiology; Kidney; Magnetic Resonance Imaging

\section{ARTICLE INFO}

Int Braz J Urol. 2017; 43: 987-9

Submitted for publication:

December 06, 2016

Accepted after revision:

January 24, 2017

Published as Ahead of Print: June 01, 2017

\section{CASE PRESENTATION}

A 53-year-old man underwent computed tomography (CT) for renal stone evaluation. His physical examination was otherwise unremarkable. His creatinine level was $1.0 \mathrm{mg} / \mathrm{dL}$ and his fasting glucose was $91 \mathrm{mg} / \mathrm{dL}$. An incidental left renal mass was identified (Figure-1), that was further evaluated with magnetic resonance imaging (MRI).

MRI showed a renal mass with thick septa and progressive enhancement after gadolinium injection. The lesion was interpreted as a complex renal cystic lesion, classified as Bosniak IV (Figures 2-5).

After MRI results, patient underwent videolaparoscopic resection of the lesion, later confirmed to be a renal anastomosing hemangioma by histopathological analysis (Figure-6).

\section{DISCUSSION}

Renal vascular tumors are extremely rare, with hemangiomas being the most frequent lesion in this subgroup (1).

The vast majority of renal hemangiomas are smaller than $2 \mathrm{~cm}$, asymptomatic and incidentally found on imaging exams. Symptomatic patients may have recurrent episodes of hematuria and abdominal pain $(1,2)$.

The anastomosing hemangioma is a rare variant, which histologically simulates an angiosarcoma (3). This histological subtype has been recently described as morphological variant of hemangioma that occurs primarily in the genitourinary tract. On non-enhanced CT, they are lobulated lesions, with soft-tissue attenuation. After contrast administration, they appear as 
Figure 1 - Corticomedullary phase from the urotomography demonstrates the lesion (arrow) with wall and septa enhancement.

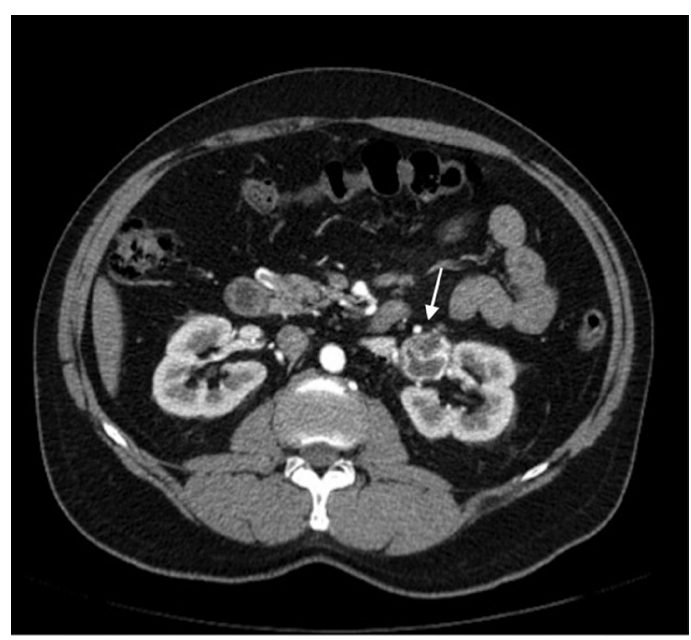

Figure 2 - Axial T2 imaging with fat saturation shows an expansive, exophytic lobulated mass with high signal, (arrow) in the upper pole left kidney.

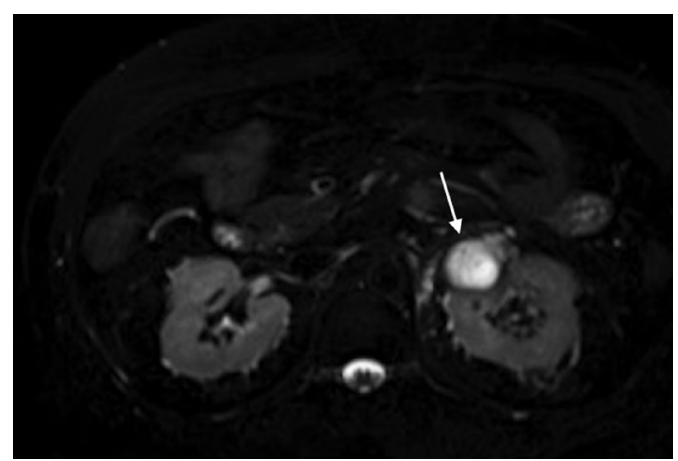

Figure 3 - Coronal T1 pre-contrast imaging - the lesion is hypointense to adjacent renal parenchyma (arrow).

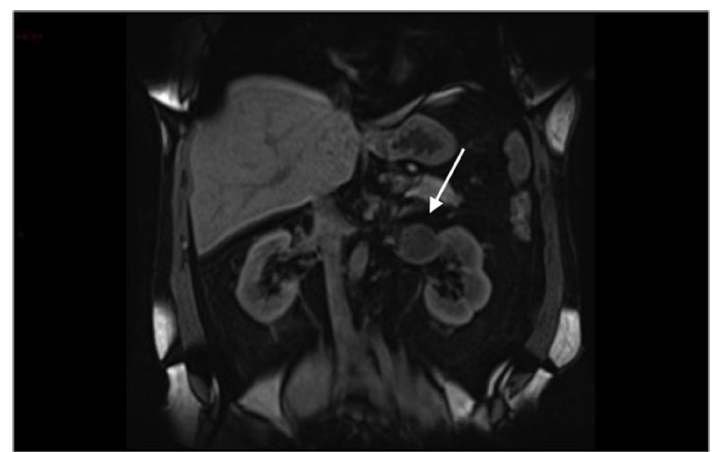

Figure 4 - Coronal T1 post-contrast arterial phase imaging showing peripheral enhancement (arrow).

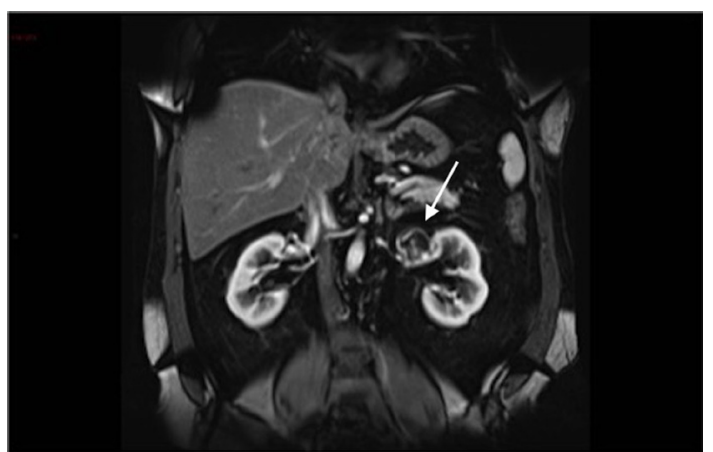

Figure 5 - Coronal T1 late post-contrast phase shows progressive enhancement of the lesion.

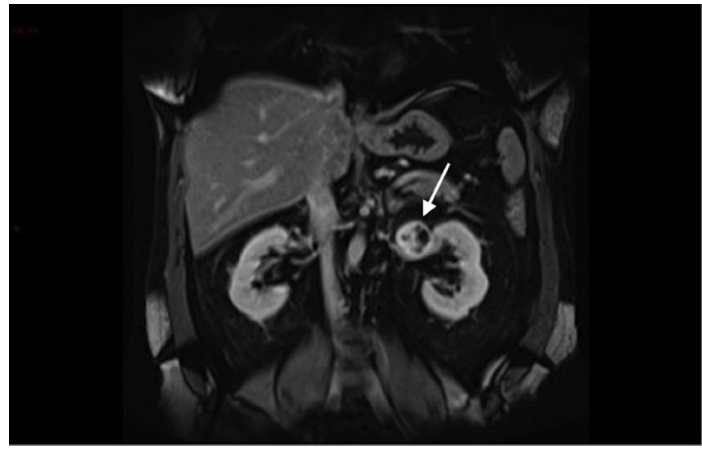

Figure 6 - Histologic sample of the ressected lesion shows anastomosing proliferation of capillary sized vessels, reminiscent of splenic sinusoids and scattered hobnailed endothelial cells, confirming the diagnosis of an anastomosing hemangioma

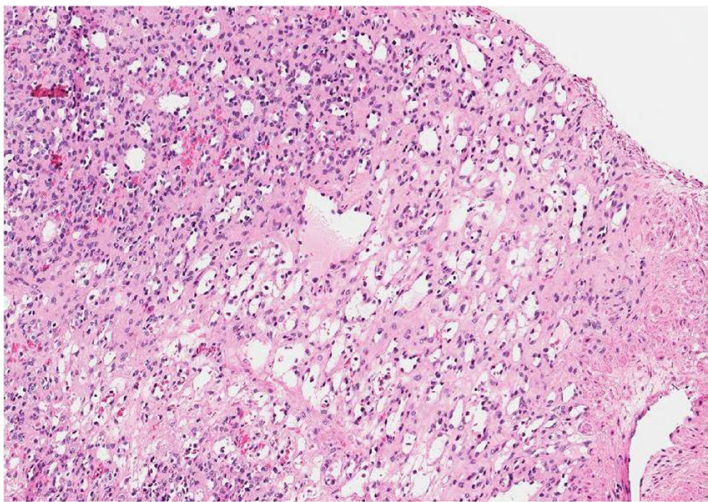


solid heterogeneous lesions, with intense and progressive enhancement (3).

On MRI, hemangiomas show hyperintensity on T2 and variable degrees of enhancement after contrast administration. Presentations may resemble cystic lesions with solid component, mimicking cystic renal cell carcinoma as the present case $(1,2,4)$. When large, these lesions are indistinguishable from malignant lesions such as angiosarcomas and renal cell carcinomas with central necrosis.

Treatment is controversial since preoperative diagnosis is not possible based on imaging exams. When biopsy results are available, it may vary from expectation to partial nephrectomy, embolization and radical nephrectomy, depending on the lesion size, location and presence of symptoms.

\section{REFERENCES}

1. Katabathina VS, Vikram R, Nagar AM, Tamboli P, Menias CO, Prasad SR. Mesenchymal neoplasms of the kidney in adults: imaging spectrum with radiologic-pathologic correlation. Radiographics. 2010;30:1525-40.

2. Prasad SR, Surabhi VR, Menias CO, Raut AA, Chintapalli KN Benign renal neoplasms in adults: cross-sectional imaging findings. AJR Am J Roentgenol. 2008;190:158-64.
3. Omiyale AO. Anastomosing hemangioma of the kidney: a literature review of a rare morphological variant of hemangioma. Ann Transl Med. 2015;3:151.

4. Tao LL, Dai Y, Yin W, Chen J. A case report of a renal anastomosing hemangioma and a literature review: an unusual variant histologically mimicking angiosarcoma. Diagn Pathol. 2014;9:159.

Correspondence address: Eduardo Kaiser Ururahy Nunes Fonseca, MD Departamento de Imagem, Hospital Israelita Albert Einstein Av. Albert Einstein, 627 São Paulo, SP, 05652-901, Brasil Fax: + 5511 2151-0195 E-mail: edukaiser_unf@hotmail.com 